\title{
Weight Identification of a Weighted Bipartite Graph Complex Dynamical Network with Coupling Delay
}

\author{
Zhen Jia and Guangming Deng \\ College of Science, Guilin University of Technology, Guilin 541004, China \\ Correspondence should be addressed to Zhen Jia, jjjzzz0@163.com
}

Received 25 March 2010; Accepted 16 July 2010

Academic Editor: Alexander I. Domoshnitsky

Copyright (c) 2010 Z. Jia and G. Deng. This is an open access article distributed under the Creative Commons Attribution License, which permits unrestricted use, distribution, and reproduction in any medium, provided the original work is properly cited.

We propose a network model, a weighted bipartite complex dynamical network with coupling delay, and present a scheme for identifying the weights of the network. Based on adaptive synchronization technique, weight trackers are designed for identifying the edge weights between nodes of the network by monitoring the dynamical evolution of the synchronous networks with drive-response structure. The conclusion is proved theoretically by Lyapunovs stability theory and LaSalle's invariance principle. Compared with the similar works, taking into consideration the structural characteristics of the network, the tracking devices designed in our paper are more effective and more easy to implement. Finally, numerical simulations show the effectiveness of the proposed method.

\section{Introduction}

Since the discoveries of the small-world (SW) [1] and scale-free (SF) [2] properties, complex networks have been studied intensively in various disciplines, such as social, biological, mathematical, and engineering sciences [3]. Synchronization is one of the most common dynamical processes and a typical collective behavior in networks. In recent years, many existing literatures devoted to the synchronization of complex dynamical networks provided with certain topology, such as SW, SF, and ring or chain networks [4-9]. However, the topology (or edge weight) of many realistic networks is uncertain or unknown. Study shows that the topological structure and edge weight directly affect the synchronous ability of networks [10]. Therefore, it is very important significance to identify the topology or estimate the edge weight in the research of complex networks. Very recently, topology identification of complex dynamical networks has been intensively studied [11-14]. The study in [11] 
suggested a method for estimating the adjacency matrix of networks with various oscillators. In $[12,13]$, the authors have provided methods to identify the topology for general networks and delay coupled networks, respectively. The study in [14] has further investigated the key factor, the independent condition, for guaranteeing successful topology identification, and it pointed out that the earlier results in [11-13] were incomplete or incorrect. The topology identification process based on [11-13] may fail due to the lack of "independent condition". Now, for a special network, such as a bipartite graph network proposed below, it is worth of further study how to design more suitable and more effective controllers to guarantee the topology or weight identification utilizing the structural feature of the network.

Bipartite graph networks widely exist in biological, social, physical, and technological fields. The so-called bipartite graph refers to a graph which has two types of nodes and edges running only between nodes of unlike types [15]. Many social and biological networks are bipartite. For example, in the research of human disease genomics, if it regards various human diseases as a type of nodes and pathogenic genes as another, human diseases and pathogenic genes make up a bipartite graph network [16]. Obviously, it is very important to identify the relation of the two classes of nodes for helping people to treat diseases. So, the research of the edge weight between nodes in a bipartite graph network has the widespread practical significance and the application value.

Motivated by the above discussions, in this paper, we provide a weighted bipartite complex dynamical network model and focus on the weight identifying problem. Based on adaptive synchronization technique, we design trackers to identify the edge weights of the network. The conclusion is proved rigorously by LaSalle's invariance principle, and a numerical example with the chaotic Lorenz system and the Chen system is provided to demonstrate the effectiveness of the proposed method.

In the whole paper, $\|\cdot\|$ represents 2-norm of vector, $(\cdot)^{T}$ denotes the transposition of $(\cdot), \otimes$ represents the Kronecker product, $I_{m}$ is an $m$-order identity matrix, and $N_{1}^{s}$ denotes the set $\{1,2, \ldots, s\}$.

\section{Model Description and Preliminaries}

Consider a weighted bipartite graph complex dynamical network with delay linear coupling, which consists by two different types of nodes, as described below:

$$
\begin{aligned}
& \dot{x}_{i}(t)=f\left(t, x_{i}(t)\right)+\sum_{j=1}^{r} p_{i j} A\left(y_{j}(t-\tau)-x_{i}(t-\tau)\right), \quad i \in N_{1}^{s}, \\
& \dot{y}_{j}(t)=g\left(t, y_{j}(t)\right)+\sum_{i=1}^{s} p_{i j} A\left(x_{i}(t-\tau)-y_{j}(t-\tau)\right), \quad j \in N_{1}^{r},
\end{aligned}
$$

where $x_{i}(t), y_{j}(t) \in R^{n}$ are the state vectors of nodes, $f, g: R^{+} \times R^{n} \rightarrow R^{n}$ are continuously differentiable vector functions. The two sets of node equation are described by $\dot{x}(t)=$ $f(t, x(t))$ and $\dot{y}(t)=g(t, y(t))$, and $s, r$ represent the number of two types of nodes, respectively. $\tau>0$ is a constant for the coupling delay. $A \in R^{n \times n}$ is a constant matrix called inner-coupling matrix. $P=\left(p_{i j}\right)_{s \times r}$ represents an unknown or uncertain coupling weight matrix, in which $p_{i j} \neq 0$ if there is a coupling from node $i$ to node $j$, and $p_{i j}$ represents the edge weight; otherwise, $p_{i j}=0$. The topology and weight information of the network connections 
is determined by the weight matrix $P$. The external-coupling matrix of network (2.1) is given by

$$
C=\left(c_{i j}\right)=\left[\begin{array}{cc}
D_{1} & P \\
P^{T} & D_{2}
\end{array}\right] \in R^{(s+r) \times(s+r)}
$$

where $D_{1}=\operatorname{diag}\left(-\sum_{j=1}^{r} p_{1 j}, \ldots,-\sum_{j=1}^{r} p_{s j}\right) \in R^{s \times s}$ and $D_{2}=\operatorname{diag}\left(-\sum_{i=1}^{s} p_{i 1}, \ldots,-\sum_{i=1}^{s} p_{i r}\right) \in$ $R^{r \times r}$.

Obviously, matrix $C$ is a diffusive coupling matrix which has zero-row sums; that is, $c_{i i}=-\sum_{k=1}^{s+r} c_{i k}, i \in N_{1}^{s+r}$.

Our objective is to design weight trackers to identify the weights of the network (2.1), that is, to estimate the elements of the unknown or uncertain weight matrix $P=\left(p_{i j}\right)_{s \times r}$. For this purpose, here we introduce a useful assumption and lemma.

Assumption 1 (A1). Suppose that there exist positive constants $\delta_{f}$ and $\delta_{g}$ such that

$$
\begin{aligned}
& \|f(t, x(t))-f(t, y(t))\| \leq \delta_{f}\|x(t)-y(t)\|, \\
& \|g(t, x(t))-g(t, y(t))\| \leq \delta_{g}\|x(t)-y(t)\|,
\end{aligned}
$$

where $x(t), y(t)$ are time-varying vectors.

Lemma 2.1. For any vectors $x, y \in R^{n}$, one has $2 x^{T} y \leq x^{T} x+y^{T} y$.

\section{Main Result}

Taking the network (2.1) as the drive network, a controlled response network can be designed as

$$
\begin{gathered}
\dot{\hat{x}}_{i}(t)=f\left(t, \widehat{x}_{i}(t)\right)+\sum_{j=1}^{r} \widehat{p}_{i j} A\left(\widehat{y}_{j}(t-\tau)-\widehat{x}_{i}(t-\tau)\right)+u_{i}, \quad i \in N_{1}^{s}, \\
\dot{\hat{y}}_{j}(t)=g\left(t, \widehat{y}_{j}(t)\right)+\sum_{i=1}^{s} \widehat{p}_{i j} A\left(\widehat{x}_{i}(t-\tau)-\widehat{y}_{j}(t-\tau)\right)+u_{s+j}, \quad j \in N_{1}^{r},
\end{gathered}
$$

where $\widehat{x}_{i}(t), \widehat{y}_{j}(t) \in R^{n}$ are the response state vectors, $u_{i}$ and $u_{s+j}$ are the control inputs to be designed, and $\hat{p}_{i j}$ is the estimation of the weight $p_{i j}$. The synchronous error between systems (2.1) and (3.1) is defined as $e_{i}(t)=\widehat{x}_{i}(t)-x_{i}(t)$ and $e_{s+j}(t)=\widehat{y}_{j}(t)-y_{j}(t), i \in N_{1}^{s}, j \in N_{1}^{r}$. 
Denote that $e(t)=\left(e_{1}^{T}(t), \ldots, e_{s}^{T}(t), e_{s+1}^{T}(t), \ldots, e_{s+r}^{T}(t)\right)^{T}$, and $\tilde{p}_{i j}=\hat{p}_{i j}-p_{i j}$. Then the error system can be written as follows:

$$
\begin{aligned}
\dot{e}_{i}(t)= & f\left(t, \widehat{x}_{i}(t)\right)-f\left(t, x_{i}(t)\right)+\sum_{j=1}^{r} \tilde{p}_{i j} A\left(y_{j}(t-\tau)-x_{i}(t-\tau)\right) \\
& +\sum_{j=1}^{r} \hat{p}_{i j} A\left(e_{s+j}(t-\tau)-e_{i}(t-\tau)\right)+u_{i}, \quad i \in N_{1}^{s}, \\
\dot{e}_{S+j}(t)= & g\left(t, \widehat{y}_{j}(t)\right)-g\left(t, y_{j}(t)\right)+\sum_{i=1}^{s} \tilde{p}_{i j} A\left(x_{i}(t-\tau)-y_{j}(t-\tau)\right) \\
& +\sum_{i=1}^{s} \hat{p}_{i j} A\left(e_{i}(t-\tau)-e_{s+j}(t-\tau)\right)+u_{s+j}, \quad j \in N_{1}^{r},
\end{aligned}
$$

or

$$
\begin{aligned}
\dot{e}_{i}(t)= & f\left(t, \widehat{x}_{i}(t)\right)-f\left(t, x_{i}(t)\right)+\sum_{j=1}^{r} \tilde{p}_{i j} A\left(\widehat{y}_{j}(t-\tau)-\widehat{x}_{i}(t-\tau)\right) \\
& +\sum_{j=1}^{r} p_{i j} A\left(e_{s+j}(t-\tau)-e_{i}(t-\tau)\right)+u_{i}, \quad i \in N_{1}^{s}, \\
\dot{e}_{S+j}(t)= & g\left(t, \widehat{y}_{j}(t)\right)-g\left(t, y_{j}(t)\right)+\sum_{i=1}^{s} \tilde{p}_{i j} A\left(\widehat{x}_{i}(t-\tau)_{i}-\widehat{y}_{j}(t-\tau)\right) \\
& +\sum_{i=1}^{s} p_{i j} A\left(e_{i}(t-\tau)-e_{s+j}(t-\tau)\right)+u_{s+j}, \quad j \in N_{1}^{r},
\end{aligned}
$$

where (3.2) and (3.3) are equivalent.

Theorem 3.1. Suppose that A1 holds. Take the controller and adaptive laws as follows

$$
\begin{gathered}
u_{i}=-k_{i} e_{i}(t), \quad \dot{k}_{i}=e_{i}^{T}(t) e_{i}(t), \quad i \in N_{1}^{s+r}, \\
\dot{\hat{p}}_{i j}(t)=\left(e_{s+j}(t)-e_{i}(t)\right)^{T} A\left(\widehat{y}_{j}(t-\tau)-\widehat{x}_{i}(t-\tau)\right), \quad i \in N_{1}^{s}, j \in N_{1}^{r},
\end{gathered}
$$

Then one has $e(t) \rightarrow 0(t \rightarrow+\infty)$; that is, the systems (2.1) and (3.1) achieve synchronization. Furthermore, if vectors $y_{1}(t)-x_{i}(t), y_{2}(t)-x_{i}(t), \ldots$, and $y_{r}(t)-x_{i}(t)\left(i \in N_{1}^{s}\right)$ or vectors $x_{1}(t)-$ $y_{j}(t), x_{2}(t)-y_{j}(t), \ldots$, and $x_{s}(t)-y_{j}(t)\left(j \in N_{1}^{r}\right)$ are linear independence, then one has $\tilde{p}_{i j} \rightarrow 0$, that is, $\hat{p}_{i j} \rightarrow p_{i j}$ as $t \rightarrow+\infty$.

Proof. Choose the Lyapunov candidate as

$$
V(t)=\frac{1}{2} \sum_{i=1}^{s+r} e_{i}^{T}(t) e_{i}(t)+\frac{1}{2} \sum_{i=1}^{s} \sum_{j=1}^{r} \tilde{p}_{i j}^{2}+\frac{1}{2} \sum_{i=1}^{s+r}\left(k_{i}-k\right)^{2}+\frac{1}{2} \int_{t-\tau}^{t} \sum_{i=1}^{s+r} e_{i}^{T}(\zeta) e_{i}(\zeta) d \zeta,
$$

where $k$ is a positive constant to be determined. 
The derivative of $V(t)$ along the trajectories of (3.3), (3.4), and (3.5) is given by

$$
\begin{aligned}
& \dot{V}(t)=\sum_{i=1}^{s} e_{i}^{T}(t) \dot{e}_{i}(t)+\sum_{j=1}^{r} e_{s+j}^{T}(t) \dot{e}_{s+j}(t)+\sum_{i=1}^{s} \sum_{j=1}^{r} \widetilde{p}_{i j} \dot{\bar{p}}_{i j}+\sum_{i=1}^{s+r}\left(k_{i}-k\right) \dot{k}_{i} \\
& +\frac{1}{2} \sum_{i=1}^{s+r} e_{i}^{T}(t) e_{i}(t)-\frac{1}{2} \sum_{i=1}^{s+r} e_{i}^{T}(t-\tau) e_{i}(t-\tau) \\
& =\sum_{i=1}^{s} e_{i}^{T}(t)\left[f\left(t, \widehat{x}_{i}(t)\right)-f\left(t, x_{i}(t)\right)\right]+\sum_{i=1}^{s} \sum_{j=1}^{r} e_{i}^{T}(t) \tilde{p}_{i j} A\left(\widehat{y}_{j}(t-\tau)-\widehat{x}_{i}(t-\tau)\right) \\
& +\sum_{i=1}^{s} \sum_{j=1}^{r} e_{i}^{T}(t) p_{i j} A\left(e_{s+j}(t-\tau)-e_{i}(t-\tau)\right)+\sum_{i=1}^{S} e_{i}^{T} u_{i}+\sum_{j=1}^{r} e_{s+j}^{T}(t)\left[g\left(t, \widehat{y}_{j}(t)\right)-g\left(t, y_{j}(t)\right)\right] \\
& +\sum_{i=1}^{s} \sum_{j=1}^{r} e_{s+j}^{T}(t) \tilde{p}_{i j} A\left(\widehat{x}_{i}(t-\tau)-\widehat{y}_{j}(t-\tau)\right)+\sum_{i=1}^{s} \sum_{j=1}^{r} e_{s+j}^{T}(t) p_{i j} A\left(e_{i}(t-\tau)-e_{s+j}(t-\tau)\right) \\
& +\sum_{j=1}^{r} e_{s+j}^{T}(t) u_{s+j}+\sum_{i=1}^{s} \sum_{j=1}^{r} \tilde{p}_{i j} \dot{\hat{p}}_{i j}+\sum_{i=1}^{s+r}\left(k_{i}-k\right) \dot{k}_{i}+\frac{1}{2} \sum_{i=1}^{s+r} e_{i}^{T}(t) e_{i}(t)-\frac{1}{2} \sum_{i=1}^{s+r} e_{i}^{T}(t-\tau) e_{i}(t-\tau) \\
& \leq \delta_{f} \sum_{i=1}^{s}\left\|e_{i}\right\|^{2}+\sum_{i=1}^{s} \sum_{j=1}^{r} p_{i j}\left[e_{i}^{T}(t) A\left(e_{S+j}(t-\tau)-e_{i}(t-\tau)\right)+e_{s+j}^{T}(t) A\left(e_{i}(t-\tau)-e_{S+j}(t-\tau)\right)\right] \\
& +\delta_{g} \sum_{j=1}^{r}\left\|e_{s+j}\right\|^{2}+\sum_{i=1}^{s} \sum_{j=1}^{r} \widetilde{p}_{i j}\left[e_{i}^{T}(t) A\left(\widehat{y}_{j}(t-\tau)-\widehat{x}_{i}(t-\tau)\right)\right. \\
& \left.+e_{s+j}^{T}(t) A\left(\widehat{x}_{i}(t-\tau)-\widehat{y}_{j}(t-\tau)\right)+\dot{\hat{p}}_{i j}\right] \\
& +\sum_{i=1}^{s+r} e_{i}^{T}(t) u_{i}+\sum_{i=1}^{s+r}\left(k_{i}-k\right) e_{i}^{T}(t) e_{i}(t)+\frac{1}{2} e^{T}(t) e(t)-\frac{1}{2} e^{T}(t-\tau) e(t-\tau) \\
& =\delta_{f} \sum_{i=1}^{s} e_{i}^{T}(t) e_{i}(t)+\delta_{g} \sum_{j=1}^{r} e_{s+j}^{T}(t) e_{s+j}(t) \\
& +\sum_{i=1}^{s} \sum_{j=1}^{r} p_{i j}\left[e_{i}^{T}(t) A\left(e_{s+j}(t-\tau)-e_{i}(t-\tau)\right)+e_{s+j}^{T}(t) A\left(e_{i}(t-\tau)-e_{s+j}(t-\tau)\right)\right] \\
& -k e^{T}(t) e(t)+\frac{1}{2} e^{T}(t) e(t)-\frac{1}{2} e^{T}(t-\tau) e(t-\tau) .
\end{aligned}
$$

because

$$
\begin{aligned}
\sum_{i=1}^{S} \sum_{j=1}^{r} p_{i j}\left[e_{i}^{T}(t) A\left(e_{s+j}(t-\tau)-e_{i}(t-\tau)\right)+e_{s+j}^{T}(t) A\left(e_{i}(t-\tau)-e_{S+j}(t-\tau)\right)\right] \\
=\sum_{i=1}^{s} \sum_{j=1}^{r} e_{i}^{T}(t) p_{i j} A e_{s+j}(t-\tau)+\sum_{i=1}^{s} \sum_{j=1}^{r} e_{s+r}^{T}(t) p_{i j} A e_{i}(t-\tau) \\
\quad+\sum_{i=1}^{S} e_{i}^{T}(t) c_{i i} A e_{i}(t-\tau)+\sum_{j=1}^{r} e_{s+j}^{T}(t) c_{s+j, s+j} A e_{S+j}(t-\tau) \\
=e^{T}(t)(C \otimes A) e(t-\tau) \triangleq e^{T}(t) G e(t-\tau),
\end{aligned}
$$


where $G=C \otimes A$. By Lemma 2.1, one has

$$
e^{T}(t) G e(t-\tau) \leq \frac{1}{2} e^{T}(t) G G^{T} e(t)+\frac{1}{2} e^{T}(t-\tau) e(t-\tau) .
$$

Therefore,

$$
\begin{aligned}
\dot{V}(t) & \leq \delta_{f} \sum_{i=1}^{s} e_{i}^{T}(t) e_{i}(t)+\delta_{g} \sum_{j=1}^{r} e_{s+j}^{T}(t) e_{s+j}(t)-k e^{T}(t) e(t)+\frac{1}{2} e^{T}(t) G G^{T} e(t)+\frac{1}{2} e^{T}(t) e(t) \\
& \leq\left(\lambda_{\max }\left(Q+\frac{1}{2} G G^{T}\right)+\frac{1}{2}-k\right) e^{T}(t) e(t)
\end{aligned}
$$

in which $Q=\operatorname{diag}\left\{\delta_{f} I_{s n}, \delta_{g} I_{r n}\right\}$. Taking $k=\lambda_{\max }\left(Q+(1 / 2) G G^{T}\right)+3 / 2$, then one has $\dot{V}(t) \leq$ $-e^{T}(t) e(t)$.

It is obvious that $\dot{V}=0$ if and only if $e(t)=0$. Let $S$ be the set of all points where $\dot{V}=0$, that is, $S=\{\dot{V}=0\}=\{e(t)=0\}$. From (3.2), the largest invariant set of $S$ is $M=$ $\left\{e(t)=0, \sum_{j=1}^{r} \tilde{p}_{i j} A\left(y_{j}(t)-x_{i}(t)\right)=0, \sum_{i=1}^{s} \tilde{p}_{i j} A\left(x_{i}(t)-y_{j}(t)\right)=0\right\}$. According to LaSalle's invariance principle [17], starting with any initial values, the trajectories of systems (3.2)(3.5) will converge to $M$ asymptotically, which implies that $e(t) \rightarrow 0(t \rightarrow+\infty)$. By the linear independence condition in Theorem 3.1, $\sum_{j=1}^{r} \tilde{p}_{i j} A\left(y_{j}(t)-x_{i}(t)\right)=0$, and $\sum_{i=1}^{s} \tilde{p}_{i j} A\left(x_{i}(t)-\right.$ $\left.\left.y_{j}(t)\right)=0\right\}$, we can get $\tilde{p}_{i j}=0$. Therefore, one has $\tilde{p}_{i j} \rightarrow 0$; that is, $\hat{p}_{i j} \rightarrow p_{i j}$ as $t \rightarrow+\infty$. Now the proof is completed.

Remark 3.2. By $\hat{p}_{i j} \rightarrow p_{i j}$, it is show that $\dot{\hat{p}}_{i j}=\left(e_{s+j}(t)-e_{i}(t)\right)^{T} A\left(\widehat{y}_{j}(t-\tau)-\widehat{x}_{i}(t-\tau)\right)$ is just the tracker of $p_{i j}$; that is, we can get the weight of the network by monitoring the dynamical evolution of the nodes. Here, the number of trackers is $s \times r$ which is much smaller than that of $(s+r)^{2}$ in $[12,13]$, so our method is more simple and easier to achieve.

Remark 3.3. It is noteworthy that the "linear independence condition" is very important in the identification method [14]; otherwise it may lead to identification failure. For the successful identifying, there cannot occur any synchronization between the two types of nodes in the bipartite graph network. Fortunately, the two types of nodes in a bipartite graph network generally have different dynamics; they are generally not synchronized under natural state.

\section{A Numerical Example}

To show the effectiveness of the proposed method, an illustrative example of a specific weighted bipartite graph network with coupling delay is given as follows. In network (2.1), we take the chaotic Lorenz system as one set of nodes dynamics, and the chaotic Chen system as another, and $s=2, t=3$. Assume that the inner-coupling matrix is $A=\operatorname{diag}(1,0,0)$, which implies that two sets of nodes are coupled through the first-state variable of the nodes. 

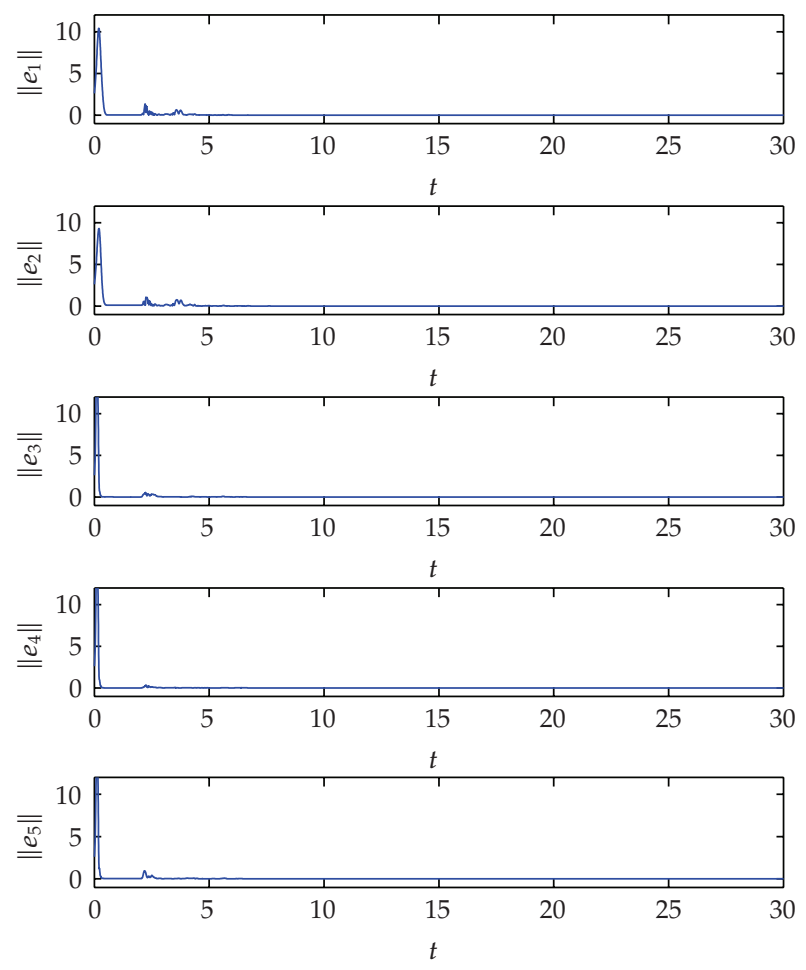

Figure 1: The evolution of the synchronous error.

The chaotic Lorenz system [18] and Chen system [19] are, respectively, described by

$$
\dot{x}_{i}=f\left(x_{i}\right)=\left[\begin{array}{c}
10\left(x_{i 2}-x_{i 1}\right) \\
28 x_{i 1}-x_{i 1} x_{i 3}-x_{i 2} \\
x_{i 1} x_{i 2}-\frac{8}{3} x_{i 3}
\end{array}\right], \quad \dot{y}_{j}=g\left(y_{j}\right)=\left[\begin{array}{c}
35\left(y_{j 2}-y_{j 1}\right) \\
-7 y_{j 1}-y_{j 1} y_{j 3}+28 y_{j 2} \\
y_{j 1} y_{j 2}-3 y_{j 3}
\end{array}\right] .
$$

Choose the coupling delay $\tau=1$ and the weight matrix

$$
P=\left[\begin{array}{rcc}
3 & 0 & -1 \\
-2 & 2 & 4
\end{array}\right]
$$

The controllers and trackers are taken as (3.4) and (3.5) in Theorem 3.1; then one can obtain the edge weights of the network: $p_{11}=3, p_{12}=0, p_{13}=-1, p_{21}=-2, p_{22}=2$, and $p_{23}=4$.

Figures 1 and 2 are the numerical simulation results. Figure 1 shows the synchronous errors that converge to zeros; that is, the response network (3.1) synchronized to the drive network (2.1). Figure 2 displays that $\hat{p}_{i j} \rightarrow p_{i j}$; that is, we have obtained the exact edge weights of network (2.1).

In the numerical simulations, the initial values are taken as follows: $x_{i}(0)=(1.5+0.5 i, 2$ $+0.5 i, 0.5 i)^{T}, y_{j}(0)=(-1.5+0.5 j, 1+0.5 j, 2.5-0.5 j)^{T}, \widehat{p}_{i j}(0)=1$, and $k_{l}(0)=1\left(l \in N_{1}^{5}\right)$. 


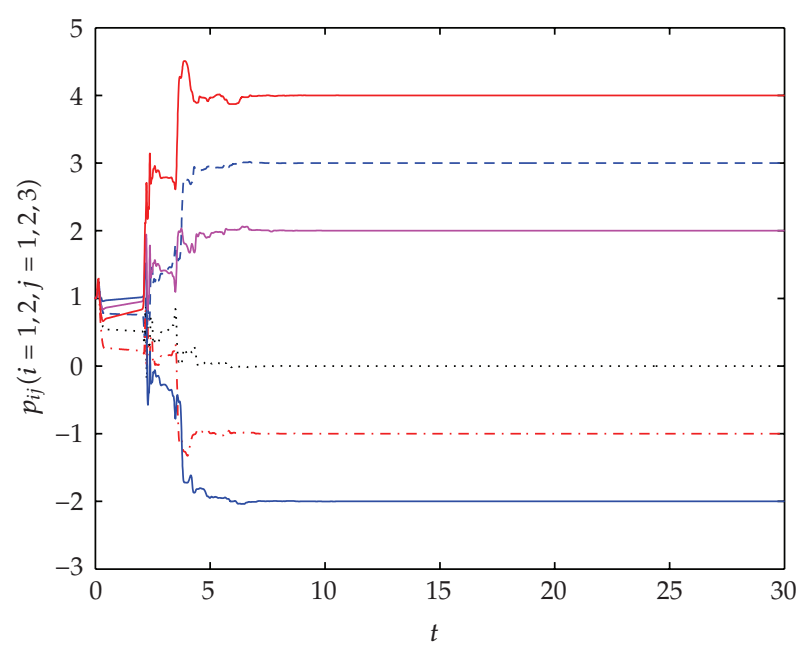

Figure 2: The evolution of the weight trackers $\widehat{p}_{i j}$.

\section{Conclusion}

In this paper, we have presented a model of weighted bipartite graph complex dynamical network with coupling delay and designed trackers for identifying the weights of the network. By monitoring the dynamical evolutions of the drive-response synchronous network, we can obtain the exact weights of the network. This approach is expected to be widely used in the study of many real bipartite graph networks, especially in the research of the relationship between two types of things.

\section{Acknowledgments}

This work was supported by the National Natural Science Foundation of China (no. 60574045), the Natural Science Foundation of Guangxi (no. 0991244) and the Science Foundation of Education Commission of Guangxi (nos. 61004101, 11061012).

\section{References}

[1] D. J. Watts and S. H. Strogatz, “Collective dynamics of 'small-world' networks," Nature, vol. 393, no. 6684, pp. 440-442, 1998.

[2] A.-L. Barabási and R. Albert, "Emergence of scaling in random networks," Science, vol. 286, no. 5439, pp. 509-512, 1999.

[3] G. R. Chen, "Introduction to complex networks and their recent advances," Advances in Mechanics, vol. 38, no. 6, pp. 653-662, 2008.

[4] A. Arenas, A. Díaz-Guilera, J. Kurths, Y. Moreno, and C. Zhou, "Synchronization in complex networks," Physics Reports, vol. 469, no. 3, pp. 93-153, 2008.

[5] J. Lü, X. Yu, and G. Chen, "Chaos synchronization of general complex dynamical networks," Physica A, vol. 334, no. 1-2, pp. 281-302, 2004.

[6] J. Lü and G. Chen, "A time-varying complex dynamical network model and its controlled synchronization criteria," IEEE Transactions on Automatic Control, vol. 50, no. 6, pp. 841-846, 2005.

[7] X. F. Wang and G. Chen, "Synchronization in small-world dynamical networks," International Journal of Bifurcation and Chaos, vol. 12, no. 1, pp. 187-192, 2002. 
[8] X. F. Wang and G. Chen, "Pinning control of scale-free dynamical networks," Physica A, vol. 310, no. 3-4, pp. 521-531, 2002.

[9] X.-P. Han and J.-A. Lu, "The changes on synchronizing ability of coupled networks from ring networks to chain networks," Science in China Series F, vol. 50, no. 4, pp. 615-624, 2007.

[10] I. Belykh, M. Hasler, M. Lauret, and H. Nijmeijer, "Synchronization and graph topology," International Journal of Bifurcation and Chaos, vol. 15, no. 11, pp. 3423-3433, 2005.

[11] D. Yu, M. Righero, and L. Kocarev, “Estimating topology of networks," Physical Review Letters, vol. 97, no. 18, Article ID 188701, 2006.

[12] J. Zhou and J.-A. Lu, "Topology identification of weighted complex dynamical networks," Physica A, vol. 386, no. 1, pp. 481-491, 2007.

[13] X. Wu, "Synchronization-based topology identification of weighted general complex dynamical networks with time-varying coupling delay," Physica A, vol. 387, no. 4, pp. 997-1008, 2008.

[14] L. Chen, J.-A. Lu, and C. K. Tse, "Synchronization: an obstacle to identification of network topology," IEEE Transactions on Circuits and Systems II, vol. 56, no. 4, pp. 310-314, 2009.

[15] M. E. J. Newman, "The structure and function of complex networks," SIAM Review, vol. 45, no. 2, pp. 167-256, 2003.

[16] K.-I. Goh, M. E. Cusick, D. Valle, B. Childs, M. Vidal, and A.-L. Barabási, "The human disease network," Proceedings of the National Academy of Sciences of the United States of America, vol. 104, no. 21, pp. 8685-8690, 2007.

[17] H. K. Khalil, Nonlinear Systems, Prentice Hall, Upper Saddle River, NY, USA, 3rd edition, 2002.

[18] E. N. Lorenz, "Deterministic non-periodic flows," Journal of Atmospheric Science, vol. 20, no. 2, pp. 130-141, 1963.

[19] G. Chen and T. Ueta, "Yet another chaotic attractor," International Journal of Bifurcation and Chaos, vol. 9, no. 7, pp. 1465-1466, 1999. 